\title{
Compound Channel's Cross-section Shape Effects on the Kinetic Energy and Momentum Correction Coefficients
}

\author{
Elham Ghanbari-Adivi \\ Assistant Professor, Department of Water Science Engineering, Shahrekord University, Shahrekord, Iran, \\ e-mail: ghanbariadivi@ @ku.ac.ir
}

(Received 26 October, 2020; revised 13 December, 2020)

\begin{abstract}
Since accurate estimation of the flow kinetic energy $(\alpha)$ and momentum $(\beta)$ is not easily possible in compound channels, determining their accurate correction coefficients is an important task. This paper has used the "flood channel facility (FCF)" data and the "conveyance estimate system (CES)" model (which is 1D, but considers a term related to the secondary flow) to study how the floodplain width and the main channel wall slope and asymmetry affect the values of $\alpha$ and $\beta$. Results have shown that their maximum values at the highest floodplain width are, respectively, 1.36 and 1.13 times of those at the lowest case; an increase in the slope increased their maximum values by 1.05 and 1.01 times, respectively. The mean of error values showed that the CES model estimated the values $\alpha$ and $\beta$ more accurately than the flow discharge. The maximum differences between the estimated and experimental values were $12.14 \%$ for $\alpha$ and $4.3 \%$ for $\beta$; for the flow discharge, it was $24.4 \%$.
\end{abstract}

Key words: CES model, compound channel, FCF, floodplain, kinetic energy correction coefficient, momentum correction coefficient

\section{Introduction}

In a compound channel, the transverse velocity distribution is broken at the interface zone because the difference of the boundary roughness and flow depths between the floodplain(s) and the main channel in this zone is high (Sellin 1964) and the non-uniform velocity distribution produces vortex flow, causing the kinetic energy to be lost and the conveyance capacity to be reduced (Keshavarzi and Hamidifar 2018).

(C) 2020 Institute of Hydro-Engineering of the Polish Academy of Sciences. This is an open access article licensed under the Creative Commons Attribution-NonCommercial-NoDerivs License (http://creativecommons.org/licenses/by-nc-nd/4.0/). 
Any deviation from the theoretical uniformity of the velocity distribution is calculated by using the kinetic energy correction (Coriolis) coefficient $(\alpha)$ and momentum correction (Boussinesq) coefficient $(\beta)$ (Mohanty 2013). Since $\alpha$ is an important factor in compound channel hydraulic calculations (Chow 1959), its careless determination will cause energy calculation errors of up to 100\% (Keshavarzi 1993). Cross-section shape, alignment, flow depth, channel slope, roughness, and so on are the factors that affect the velocity distribution for which $\alpha=\beta=1$ if it is uniform in both lateral and vertical directions (Chow 1959, French 1987). The cross-section shape affects the values of $\alpha$ and $\beta$ and their accurate calculation is essential, because if there is any negligence, estimation of the flow hydraulic parameters will be erroneous (Keshavarzi and Hamidifar 2018), resulting in a 5-10\% error in the flow calculations (Fenton 2005). In single channels, use of some predefined coefficients is acceptable, but in compound ones, accurate estimation of these coefficients will minimize the design errors (Keshavarzi and Hamidifar 2018).

In a river, if the main channel-floodplain velocity difference is high, the value of $\alpha$ can increase to more than 2 (Henderson 1966); in some related studies its value has been between 1 and 2 (Chow 1959). In their study on a symmetric smooth straight compound channel with broad floodplains, Mohanty et al (2012) have reported values of 2.09 and 1.39 for $\alpha$ and $\beta$, respectively, but Kolupaila (1956) have recommended average values of 1.75 and 1.25 for $\alpha$ and $\beta$, respectively, for over-flooded river valleys or channels fringed by floodplains. While Li and Hager (1990) suggest $\alpha=1.15$ and $\beta=1.06$ in practical applications, Seckin et al (2009a) propose $\alpha=1.156$ and $\beta=1.056$ for symmetric and asymmetric rectangular compound channels, and Parsaie (2016) recommends $\alpha=2.2$ and $\beta=1.4$ for symmetric compound channels with smooth boundaries.

A general review of the literature shows that studies on the effects of the floodplain width on the energy loss and momentum in compound sections are rare; hence, the issue is addressed here due to its importance.

\subsection{Conveyance Estimation System (CES) Model}

The CES conveyance-calculation approach is based on the depth-integrated RANS equations for flow along the stream direction. It extends the original Shiono-Knight Method (SKM) (Shiono and Knight 1989) for straight prismatic channels to include the more recent Ervine et al's approach (Ervine et al 2000) for meandering channels (CES User Manual 2004). Shiono and Knight (1991) developed a model that considered secondary flows and the depth-velocity was assumed to change linearly in the transverse direction. Called SKM (Shiono and Knight Method), it introduces a secondary flow term as $\Gamma$ in each subsection (Tang and Knight 2008). The SKM method uses the Navier-Stokes momentum equation, that presented by Shiono and Knight (1991) in a 2D equation (Shiono and Knight 1988 and 1990). 
Knight et al (1989) developed one of the first LDM (Lateral Distribution Method) models based on the Navier-Stokes-momentum equation in the flow direction for a compound channel. The classical LDM was first derived from averaging of the simple Navier-Stokes equations in depth, but the developed one is directly obtained from the simplified Saint Venant equations with dispersion terms (Bousmar 2002).

Since the existing 1D models (e.g., ISIS, HEC-RAS and MIKE 11) had unphysical base and boundary resistance, overestimated floodplains and underestimated main channels, CES was developed to estimate the conveyance by the reduced and simplified form of the Reynolds-averaged Navier-Stokes (RANS) equations (CES User Manual 2004), because it can generate parameters such as the lateral distribution of the depth-averaged velocity, boundary shear stress, transverse friction velocity and Boussinesq and Coriolis coefficients (Mohanty 2013). Studying Tisza River in Hungary, Nagy et al (2018) used Hec-Ras and CES models to find the floodplain cover effects on the flood transfer and showed that both could estimate the flood depth and velocity. To examine the transverse distribution of depth mean velocity in a meandering trapezoidal channel, Mohanty (2013) used CES to analyze the mean depth velocities and showed that the CES-estimated average velocity was less than the measured one. Studying floods in Northern Ireland to solve continuity and momentum equations of permanent flows, Moreta and Lopez-Querol (2017) used 1D Hec-Ras and CES models and SRH-2D model and concluded that CES, which calculated the flow parameters based on the LDM, estimated velocity distribution and the momentum between the main channel and the floodplain more accurately than the SRH-2D model. Singh et al (2018) applied CES and ANSYS fluent models to calculate flow parameters. They studied lateral distribution of the velocity and shear stress in a gravel-bed channel experimentally and computationally and showed that both models gave acceptable numerical results. The CES-/ANSYS-estimated shear stress distributions were, respectively, higher and lower than the experimental results; while CES provided uniform boundary shear stress at the channel bed, ANSYS 3D gave values close to the real data. Presenting a model to estimate the discharge of an asymmetric compound channel, Devi and Khatua (2019) compared its results with those of SCM, EDM, EVDM and CES and concluded that the proposed model estimated the flow rate better than CES. Devi et al (2018) used FCF data from the England Wallingford Institute and the Indian NITR Channel to study how CES estimated the mean depth of velocity in symmetric and asymmetric compound channels, and showed that although CES predicted the low-width-ratio channels accurately, it did not yield an accurate prediction in the interface zone because of uncertain values of the eddy viscosity, friction factor and secondary flow. Hence, the authors recommended correction coefficients to be used in CES, especially near the shear layer.

This study uses the FCF data to examine the effects of the floodplain width, slope of the main channel wall and asymmetry of the compound channel on the $\alpha$ and $\beta$ coefficients, and investigates the CES ability to estimate these coefficients and discharge in different conditions. The CES model which is used here is a suitable method for 
estimating the capacity of a compound channel, because it considers the effects of the secondary flow in the subsections' interface and calculates the energy loss due to the interaction between the sub-section in a compound channel, which leads to a reduced transfer capacity in these sections compared to regular ones. The FCF data that are used here are also compared with the results from other laboratory experiments. Finally, statistical methods, such as the normalized root mean square error (NRMSE) and mean absolute percentage error (MAPE) are used to determine the CES accuracy.

\section{Methods}

\subsection{Kinetic Energy and Momentum Correction Coefficients}

To find $\alpha$ and $\beta$, the compound channel is divided into several subsections; $\alpha$ is the sum of the flow kinetic energy in each subsection divided by that of the entire section (Eq. 1), and $\beta$ is the sum of momentum in each subsection divided by that of the entire section (Eq. 2) (Mohanty et al 2012). Thus,

$$
\begin{aligned}
& \alpha=\frac{\sum_{i=1}^{n} v_{i}^{3} \alpha_{1}}{V^{3} A}, \\
& \beta=\frac{\sum_{i=1}^{n} v_{i}^{2} \alpha_{1}}{V^{2} A},
\end{aligned}
$$

where $v_{i}$ and $a_{i}$ are the average velocity and area in each subsection (Fig. 1), $V$ and $A$ are those in the entire section, and $n$ is the number of subsections.

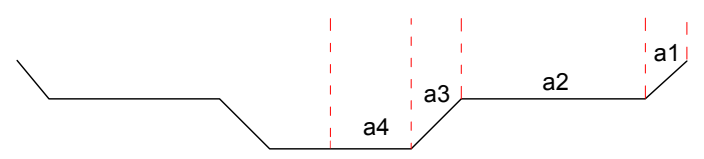

Fig. 1. Subsections of the compound channel $\left(a_{i}\right)$

As noted before, various factors (cross-section shape, flow hydraulic parameters, boundary roughness, etc.) can affect the lateral velocity distribution in a channel. This study has used the FCF data to examine the effects of the cross-section shape on $\alpha$ and $\beta$ in a channel which is $60 \mathrm{~m}$ long and $10 \mathrm{~m}$ wide, under the following three sets of conditions:

\subsubsection{Effects of the Floodplain Width}

Using Series 01, 02 and 03 (symmetric) FCF data and constant main channel bed width and wall slope, effects on $\alpha$ and $\beta$ were studied for floodplain widths of 5, 3.5 and $1.65 \mathrm{~m}$. Fig. 2 and Table 1 show the FCF series cross-section and information, respectively. 

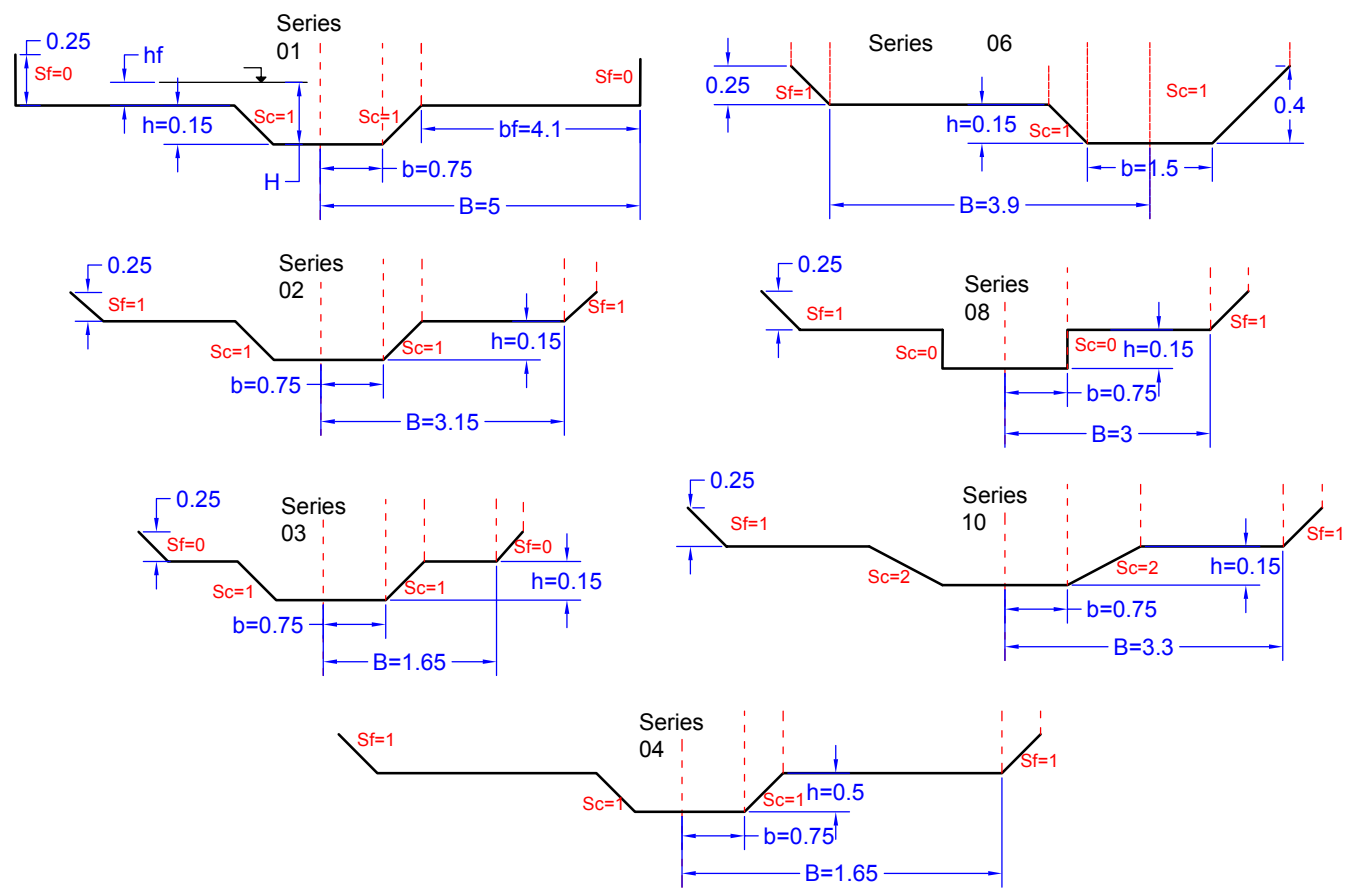

Fig. 2. FCF series cross-sections

Table 1. FCF series parameters

\begin{tabular}{|l|c|c|c|c|c|c|}
\hline Cross section Shape & $S_{c}$ & $S_{f}$ & $B$ & $b_{f}$ & $b$ & Series \\
\hline Symmetric & 1 & 0 & 5 & 4.1 & 0.75 & 01 \\
\hline Symmetric & 1 & 1 & 3.15 & 2.25 & 0.75 & 02 \\
\hline Symmetric & 1 & 1 & 1.65 & 0.75 & 0.75 & 03 \\
\hline Trapezoidal & 1 & - & - & - & 0.75 & 04 \\
\hline Asymmetric & 1 & 1 & 3.15 & 2.25 & 0.75 & 06 \\
\hline Symmetric & 0 & 1 & 3 & 2.25 & 0.75 & 08 \\
\hline Symmetric & 2 & 1 & 3.3 & 2.25 & 0.75 & 10 \\
\hline
\end{tabular}

\subsubsection{Effects of the Main Channel Wall Slope}

Using Series 02, 08 and 10 (symmetric) FCF data and constant floodplain and main channel widths, effects on $\alpha$ and $\beta$ were studied for main channel wall slopes of $1: 1$, $0: 1$ and $2: 1$.

\subsubsection{Effects of Asymmetry}

Using Series 06 (asymmetric) FCF data, effects on $\alpha$ and $\beta$ were studied for asymmetry effects.

The FCF data include the flow hydraulic parameters (discharge, area and mean velocity of the entire section and each subsection, etc.) and channel characteristics (main 
channel bed width and wall slope, floodplain width, boundary roughness and bed slope). Using the CES model, Eqs. (1) and (2) and the above-mentioned information, $\alpha$ and $\beta$ were calculated under different conditions and compared with experimental values.

In the plots, $h$ is the main channel depth, $b$ is half of the main channel width, $b_{f}$ is the floodplain width, $H$ is the main channel flow depth, $S_{f}$ is the floodplain wall slope, $S_{c}$ is the main channel wall slope and $B$ is the distance from the channel axis to the end of the floodplain, respectively.

\subsection{Model Evaluation}

Errors which were considered for evaluating the CES model accuracy to estimate $\alpha$, $\beta$ and flow discharge $Q$, included the mean absolute percentage error (MAPE) and normalized root mean square error (NRMSE), defined as follows:

$$
\begin{aligned}
\text { MAPE } & =\frac{1}{n}\left(\sum_{i=1}^{n} \frac{\left|x_{i}-y_{i}\right|}{x_{i}}\right) \times 100, \\
\text { NRMSE } & =\frac{\sqrt{\frac{1}{n} \sum_{i=1}^{n}\left(x_{i}-y_{i}\right)^{2}}}{\bar{x}} \times 100,
\end{aligned}
$$

where $x_{i}$ are the observed values ( $\alpha, \beta$ and $Q$ in the FCF data), $y_{i}$ are the estimated values ( $\alpha, \beta$ and $Q$ in the CES model), $\bar{x}$ is the mean of the observed values and $n$ is the number of values.

\section{Results and Discussion}

\subsection{Values of $\alpha$ and $\beta$ in a Single Channel}

Variations of $\alpha$ and $\beta$ calculated for a single, different flow-depth, trapezoidal channel are shown in Fig. 3.

As shown, the flow always remains in the main channel (it does not enter the floodplain) and $\alpha \approx \beta \approx 1$ for different depths because the cross-section shape is uniform, velocity vectors have the same direction (no vortex is formed) and the shear stress and momentum exchange are insignificant.

\subsection{Effects of the Floodplain Width on $\alpha$ and $\beta$}

Using Series 01, 02 and 03 of FCF data for different floodplain widths (4.1, 2.25 and $0.75 \mathrm{~m}$ ) and constant main channel wall slope resulted in $1.5 \mathrm{~m}$ for width, $0.15 \mathrm{~m}$ for depth and $1: 1$ for the main channel wall slope. Figure 4 shows the effects of the floodplain width and flow depth on the values of $\alpha$ and $\beta$. 


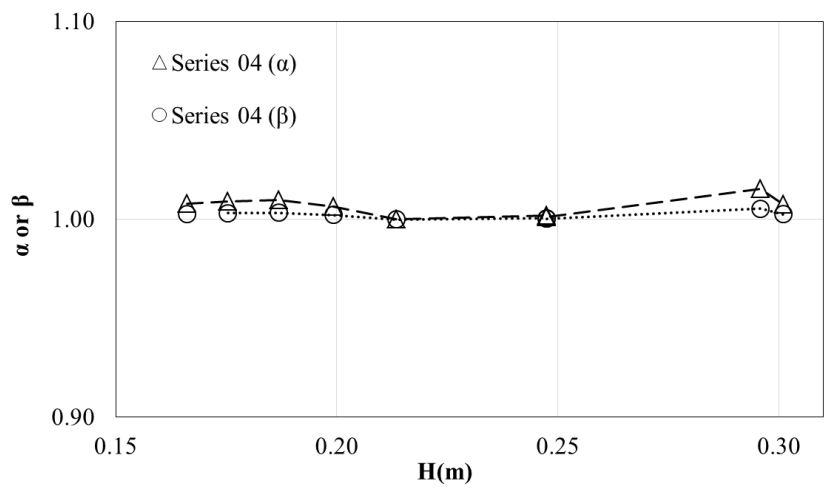

Fig. 3. Variations of $\alpha$ and $\beta$ for Series 04 of FCF data
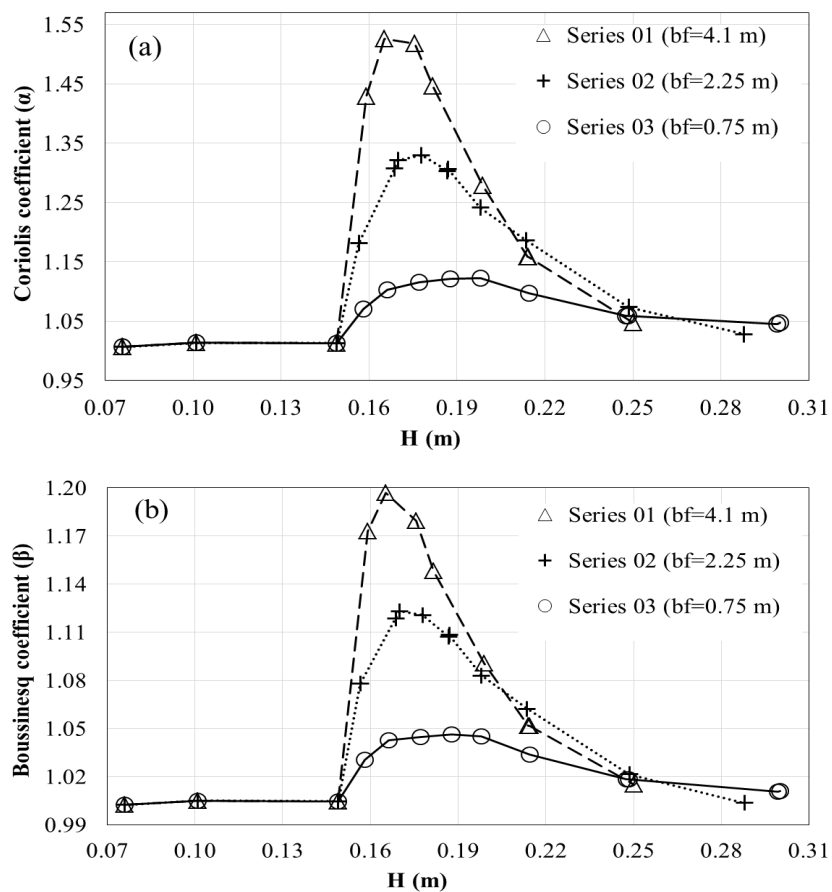

Fig. 4. Effects of the floodplain width and flow depth on $\alpha$ (a) and $\beta$ (b)

As seen in Fig. 4(a), the values of $\alpha$ and $\beta$ did not significantly change before the flow depth reached a bankfull stage $(15 \mathrm{~cm})$, but when it reached $15.8 \mathrm{~cm}$, they suddenly increased by 1.2 and 0.4 units, respectively (Parsaie 2016). The increase in the maximum $\alpha$ was $0.41,0.17$ and 0.06 in Series 01,02 and 03, respectively (i.e., the increase in Series 01 was about 2.4 times that of in Series 02, and 6.8 times of that in Series 03). Regarding $\beta$, the values were $0.17,0.08$ and 0.03 , respectively (i.e., the increase in Series 01 was about 2.1 times of that in Series 02, and 5.6 times of 
that in Series 03). Since the floodplain was wider in Series 01 compared to Series 02 and 03 , the flow in the same depth was greater, the velocity was smaller, the main channel-floodplain velocity difference was higher, the shear stress and kinetic energy loss were larger and, therefore, $\alpha$ and $\beta$ increased more. In Fig. 4(b), since the vortex flow in the interface zone was more in Series 01 than in Series 02 and 03, the main channel-floodplain momentum exchange was larger, causing $\beta$ to increase more.

An increase in the flow depth caused $\alpha$ and $\beta$ to start to decrease in all graphs, and in each series they reached their highest values at specific depths. The maximum values of $\alpha$ and $\beta$ occurring at their relative depths $D_{r}=(H-h) / H$ in Series 01, 02 and 03 were $(0.1,0.16,0.24)$ and $(0.1,0.12,0.2)$, respectively, indicating that in each series, the maximum $\beta$ occurred at a lower relative depth than that for the maximum $\alpha$. Parsaie (2016) showed that the highest $\alpha$ and $\beta$ values occurred in a $0.15-0.2 \mathrm{~m}$ flow depth range and concluded that an increase in the latter reduced $\alpha$ and $\beta$ to a minimum value $\approx 1$ in all series (Parsaie 2016 and Mohanty et al 2013, reported the same trend).

An increase in the flow depth reduced/increased the roughness/floodplain effects on the flow transfer rate causing the floodplain-main channel velocity difference to decrease, shear stress and vortex flow in the interface zone to diminish, and, thus, $\alpha$ and $\beta$ values to reduce.

The decreasing trend continued until $\alpha \approx \beta \approx 1$ and the compound channel was treated as a single one, because the effects of walls were reduced on the flow and its stream alignment. Since the maximum values of $\alpha$ and $\beta$ in Series 01, 02 and 03 are $(1.53,1.33,1.12)$ and $(1.19,1.12,1.05)$, respectively, their comparison shows notable effects of the floodplain width on their values at compound channels.

\subsection{Effects of the Main Channel Wall Slope on $\alpha$ and $\beta$}

Effects of the main channel wall slope on $\alpha$ and $\beta$ were studied using data Series 02, 08 and 10, when the floodplain width and main channel width and depth were $3.15,1.5$ and $0.15 \mathrm{~m}$, and the main channel wall slopes were $1: 1,0: 1$ and $2: 1$, respectively (Fig. 5).

In Fig. 5(a), an increase in the main channel flow depth (to more than $0.15 \mathrm{~m}$ ) suddenly increased $\alpha$ and $\beta$ to their maximum values in each series, because, as mentioned before, the flow entered the floodplain and caused some shear stress to form in the interface zone due to the floodplain-main channel flow velocity difference. This led to a kinetic energy loss and increased $\alpha$ causing the momentum exchange and, hence, $\beta$ to increase too because of the vortex flow in this area. According to the rising branch of $\alpha$ and $\beta$ curves, an increase in the flow depth reduced the branch slope, causing $\alpha$ and $\beta$ to reach their maximum values which, for $\alpha$, were equal to 1.33, 1.34 and 1.32 in Series 02, 08 and 10, respectively. Since the main channel wall slope increased from $2: 1$ in Series 10 to $1: 1$ in Series 02 and then to $0: 1$ in Series 08, the distance between the main channel and floodplain flow regions was decreased, 

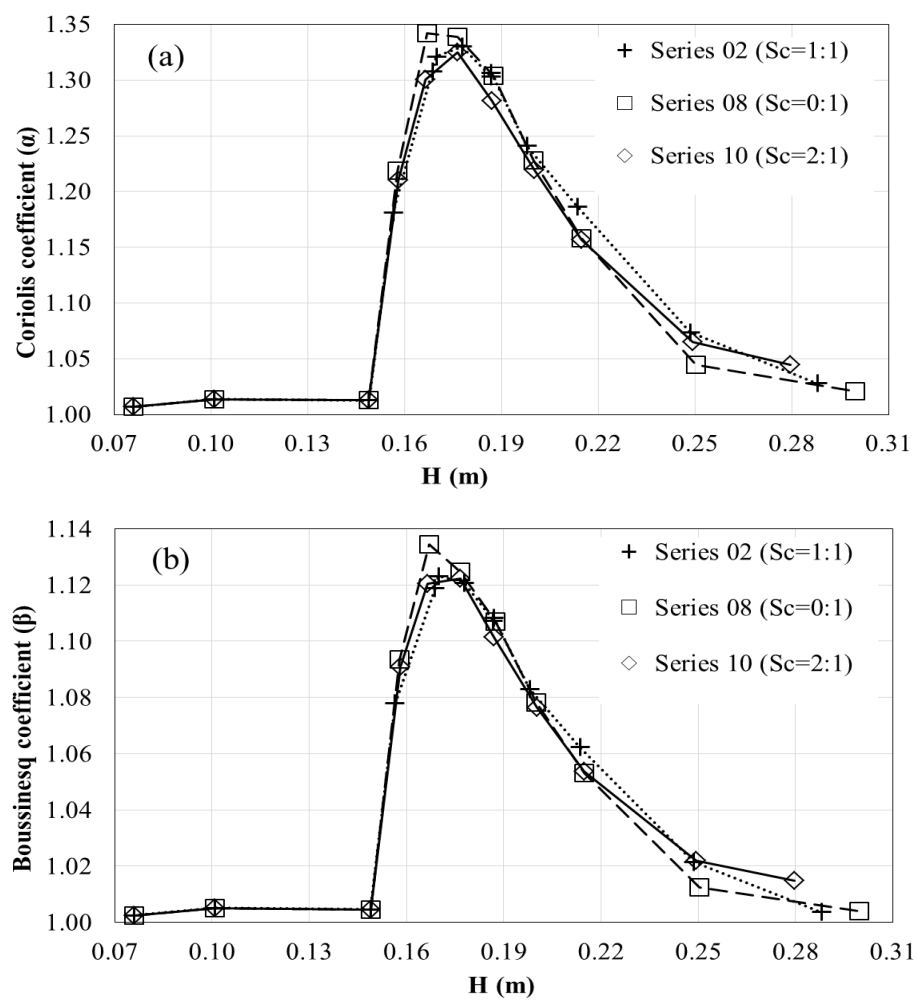

Fig. 5. Effects of the main channel wall slope and flow depth on $\alpha$ (a) and $\beta$ (b)

causing the shear stress to increase in the interface zone. Hence, an increase in the main channel wall slope slightly increased the maximum value of $\alpha$.

In Fig. 5(b), since the maximum values of $\beta$ are 1.12, 1.13 and 1.12 in Series 02, 08 and 10, respectively, the effects of the main channel wall slope on $\alpha$ and $\beta$ are negligible under these conditions. Again, an increase in the flow depth reduced the boundary roughness effects and increased the floodplain effects on the flow transfer rate, leading to a reduction in the shear stress and flow vortex, and causing $\alpha \approx \beta \approx 1$ when the compound channel behaves as a single one.

\subsection{Compound Channel's Asymmetry Effects on $\alpha$ and $\beta$}

The compound channel's asymmetry effects were examined on $\alpha$ and $\beta$ using Series 06 FCF data, when the floodplain width and main channel width, depth and wall slope were 3, 1.5 and $0.15 \mathrm{~m}$ and $1: 1$, respectively, and $\alpha$ and $\beta$ were calculated (Fig. 6) by using Eqs. (1) and (2).

In Fig. 6(a), an increase in the flow depth beyond the bankfull, as in the symmetric channel, caused a sudden increase in $\alpha$, and since the floodplain depth and flow velocity were smaller (than those of the main channel), its roughness had a more 

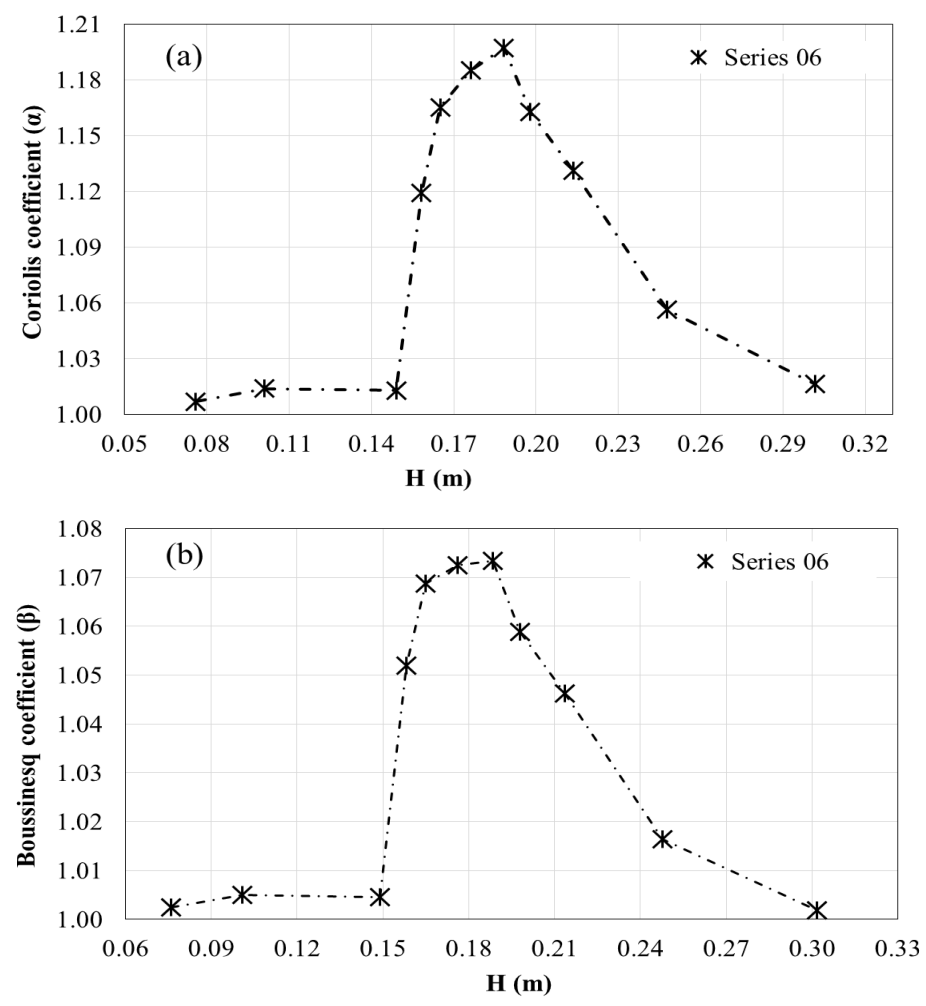

Fig. 6. Asymmetry and flow depth effects on $\alpha$ (a) and $\beta$ (b)

significant effect on the flow in this zone. The floodplain-main channel velocity difference generated some shear stress in the interface zone and the flow energy loss and non-uniform velocity distribution increased $\alpha$ (its maximum value was smaller in the asymmetric than in the symmetric channel). Further increase in the flow depth made $\alpha=1$, causing the compound channel to act as a single one. An increase in the flow depth reduced the floodplain roughness effects on the flow, causing the velocity to increase in this zone. Hence, the floodplain-main channel velocity difference was reduced, leading to a reduction in $\alpha$.

In Series 03 (with smaller floodplain width) and 01 and 02 (with higher ones), the maximum $\alpha$ was smaller in the asymmetric than in the symmetric channel, because the shear stress and kinetic energy loss were larger in the latter as there were in the two interface zones between the main channel and the floodplains, and it was smaller in the former, because there was one interface zone, concluding that $\alpha$ is affected less by the floodplain width and more by the shear stress in the interface zone.

In Fig. 6(b), the maximum $\beta$ is 1.07 for the asymmetric channel (only smaller than those in Series 01 and 02 of the symmetric channels), because, as mentioned before, its values were 1.2, 1.12 and 1.04 in Series 01, 02 and, 03 of the symmetric channels, respectively. Since the floodplain width in Series 06 was smaller than those in Series 
01 and 02, and greater than that in Series 03, it can be concluded that $\beta$ is affected less by the shear stress in the interface zone and more by the floodplain width.

\subsection{Comparison with other Studies}

The values of $\alpha$ and $\beta$ from this study were compared with those from other studies for the case of the symmetric compound channel (Fig. 7).
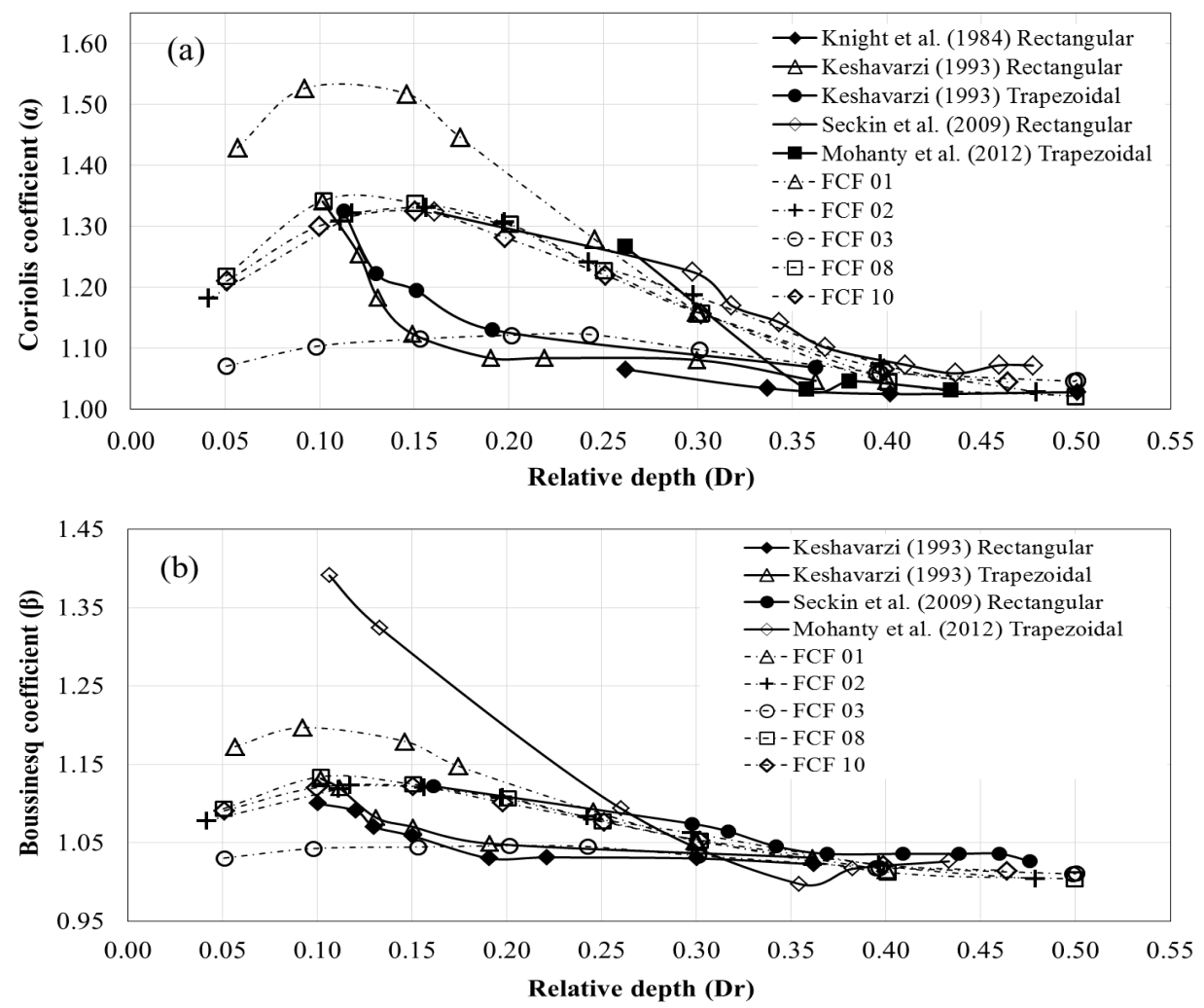

Fig. 7. Comparison of $\alpha$ and $\beta$ of this study with other studies

In this study, $\alpha$ and $\beta$ variations had a maximum and two ascending and descending branches; in the latter, $\alpha \approx \beta \approx 1$ for flow depths $>25 \mathrm{~cm}\left(D_{r}>0.4\right)$, especially for the symmetric channels, and the compound channel behaved like a single one. While Mohanty (2013) observed that $\alpha \approx \beta \approx 1$ for $D_{r}>0.35$, Seckin et al (2009b) stated that for $D_{r}>0.5$ a compound channel behaved like a single one.

\subsection{Comparison of CES Results with Experimental Data}

Series $01,02,03,06,08$ and 10 of the FCF data were simulated by the CES model to predict the values of $\alpha, \beta$ and the flow discharge $Q$. Fig. 8 compares the experimental 
and estimated results, and Table 2 contains a summary of different statistical values for $\alpha, \beta$ and the flow discharge simulated by the CES model.
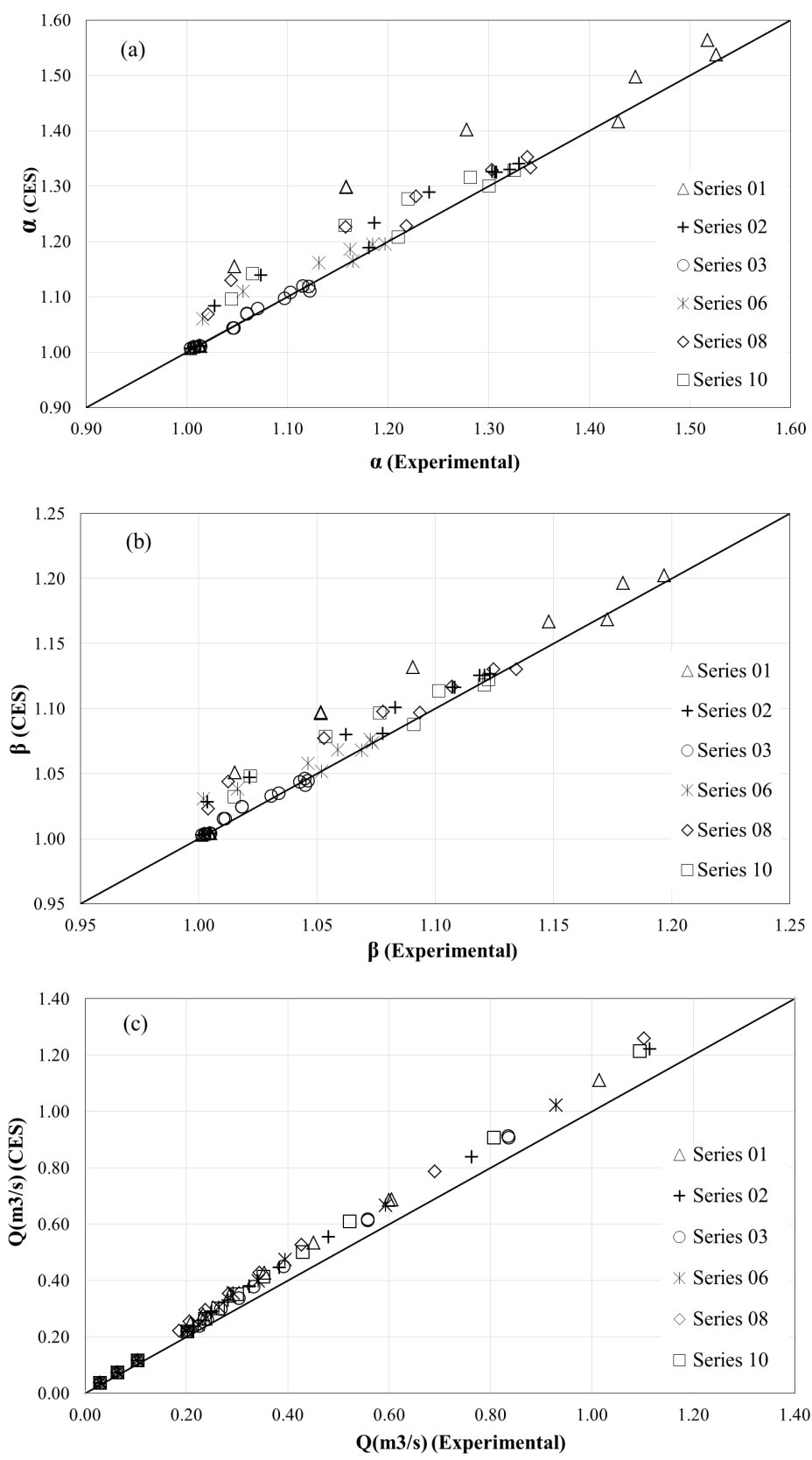

Fig. 8. Scatter diagram for the experimental and estimated values of $\alpha$ (a), $\beta$ (b) and flow discharge (c) 
Table 2. Summary of various error and $\mathrm{R} 2$ values of $\alpha, \beta$ and flow discharge simulated by the CES model

\begin{tabular}{|c|c|c|c|c|c|c|c|c|}
\hline & \multirow{2}{*}{$\begin{array}{l}\text { Statistical } \\
\text { Parameters }\end{array}$} & \multicolumn{6}{|c|}{ Series } & \multirow{2}{*}{ Mean } \\
\hline & & 01 & 02 & 03 & 06 & 08 & 10 & \\
\hline & NRMSE \% & 6.33 & 2.66 & 0.56 & 2.49 & 3.93 & 4.01 & 3.33 \\
\hline \multirow[t]{2}{*}{ a } & MAPE \% & 4.55 & 1.94 & 0.46 & 1.88 & 3.42 & 3.31 & 2.59 \\
\hline & $R^{2} \%$ & 92.49 & 97.31 & 98.1 & 92.88 & 95.94 & 92.88 & 94.93 \\
\hline \multirow{3}{*}{$\beta$} & NRMSE \% & 2.33 & 1.17 & 0.33 & 1.34 & 1.62 & 1.55 & 1.39 \\
\hline & MAPE \% & 1.67 & 0.85 & 0.26 & 0.95 & 1.38 & 1.28 & 1.07 \\
\hline & $R^{2} \%$ & 93.93 & 96.9 & 97.12 & 94.13 & 97.5 & 94.7 & 95.71 \\
\hline \multirow{3}{*}{$Q$} & NRMSE \% & 17.48 & 16.93 & 12.4 & 14.88 & 20.18 & 15.12 & 16.17 \\
\hline & MAPE \% & 16.04 & 14.33 & 11.46 & 14.30 & 20.74 & 14.69 & 15.26 \\
\hline & $R^{2} \%$ & 99.74 & 99.87 & 99.92 & 99.73 & 99.77 & 99.89 & 99.82 \\
\hline
\end{tabular}

In Figs. 8(a) and 8(b), the data distribution around the 450-line is more uniform than in Fig. 8(c).

In Table 2, the values of $\alpha$ and $\beta$ estimated for Series 03 agree well with the experimental values, but an increase in the floodplain width reduces the results' accuracy, because this increase increases the turbulent flow causing the estimation of the eddy viscosity, friction velocity, secondary flow and shear stress to become difficult to perform and inaccurate, thus reducing the accuracy of the CES model results. The max and min errors for $\alpha$ and $\beta$ occur for Series 01 and 03, respectively, and those for the discharge occur for Series 08 and 03, respectively. The mean values of the statistical parameters in Table 2 show that although the CES model estimates $\alpha$ and $\beta$ more accurately than the discharge, the estimation accuracy of all three parameters is quite acceptable.

For a comparison, the differences between the estimated and experimental values of $\alpha, \beta$ and the discharge were calculated, and these results are shown in Fig. 9, where the star sign $\left(^{*}\right)$ means percent error which is the estimated value minus the experimental value divided by the experimental value, and multiplied by 100 .

As can be seen in Fig. 9(a), before the flow depth reached a bankfull stage $(15 \mathrm{~cm})$, the difference between the experimental and estimated values was nearly zero, but when the depth increased, the latter difference increased too for all series (except Series 01), and reached its maximum value at a depth $\approx 25 \mathrm{~cm}\left(D_{r}=0.4\right)$. At a depth where this difference was the highest for $\alpha(12.14 \%$ in Series 01$)$, the compound channel behaved as a single one and, then, the difference began to decrease, with a larger increase in the flow depth. Although an increase in the floodplain width increased the above-mentioned difference, the main channel wall slope had no significant effects on it. According to Fig 8(a), the CES-estimated $\alpha$ was generally higher than the experimental value.

Regarding $\beta$ (Fig. 9b), the trend was similar to that of $\alpha$ (Fig. 9a); before the flow reached a bankfull stage $(15 \mathrm{~cm})$, the difference was $\approx 0$. Then it increased and reached 

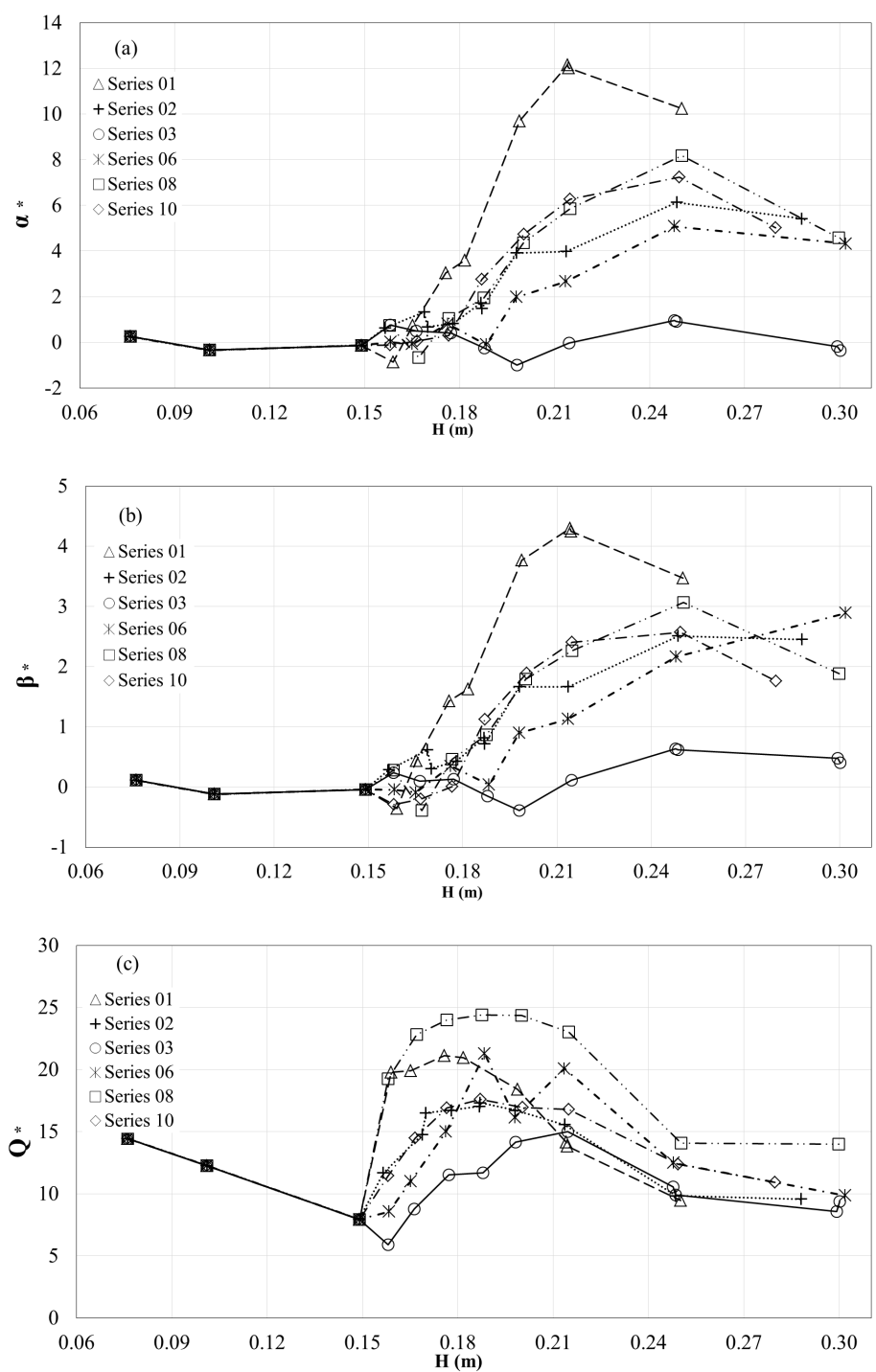

Fig. 9. Differences between the experimental and estimated values of $\alpha$ (a), $\beta$ (b) and flow discharge $Q$ (c)

its maximum (4.3\% for Series 01) when the flow depth increased. A further depth increase reduced the above-mentioned difference, the maximum of which occurred in each series at a flow depth $\approx 25 \mathrm{~cm}$. The difference had an increasing trend in Series 06, and its maximum occurred at a $21 \mathrm{~cm}$ flow depth in Series 01, when the compound channel behaved like a single one. Similarly to the $\alpha$ trend, an increase in the flood plain width increased the difference, but the main channel wall slope had no significant effects on it. According to Fig 9(b), the CES-estimated $B$ value was generally higher than from the experiments. 
According to Fig 9(c), before the flow reached a bankfull stage $(15 \mathrm{~cm})$, the difference between the experimental and estimated values of the flow discharge was reduced, but a depth increase increased this difference in all series to reach its maximum. A further flow-depth increase up to $25 \mathrm{~cm}$ (when the compound channel behaves as a single one), the difference was reduced and, after that, it remained almost constant. The highest estimated-experimental flow discharge difference was $24.4 \%$ at Series 08 .

\section{Conclusions}

In compound channels, the values of the coefficients $\alpha$ and $\beta$ suddenly increased and reached their maximum values after the flow entered the floodplain. While the maximum $\alpha$ values were $1.53,1.33$ and 1.12 in series 01,02 and 03 , those of $\beta$ were $1.19,1.12$ and 1.05 , respectively, which means that the floodplain width affects both $\alpha$ and $\beta$. The maximum $\alpha$ was always lower in an asymmetric channel (Series 06) than in symmetric ones (Series 01, 02 and 03), that of $\beta$ was higher than in symmetric channels with narrower floodplains (Series 03) and lower than in those with wider floodplains (Series 01 and 02). Therefore, in asymmetric channels, the value of $\alpha$ was less affected by the floodplain width, and was more affected by the shear stress in the interface zone, but as for $\beta$, the process was reverse.

The trend of $\alpha$ and $\beta$ variations had a maximum and two ascending and descending branches; in the latter case, $\alpha \approx \beta \approx 1$ for flow depths $>25 \mathrm{~cm}\left(D_{r}>0.4\right)$.

The estimation results of for the hydraulic flow parameters such as $\alpha, \beta$ and the flow discharge showed the high capability of the CES model in the analysis of compound channels; however, an increase in the floodplain width reduced the results' accuracy.

Based on the mean of error values, the CES model estimated the values of $\alpha$ and $\beta$ more accurately than the flow discharge, although its accuracy was acceptable for all three parameters.

Before the flow depth reached a bankfull stage $(15 \mathrm{~cm})$, the difference between the experimental and estimated values of $\alpha$ and $\beta$ was nearly zero, but when the flow depth increased, this difference increased too for all series (except Series 01 and 06), and reached its maximum value for a flow depth $\approx 25 \mathrm{~cm}$, when the compound channel behaved like a single one; the difference began to decrease with more increase in the flow depth than $25 \mathrm{~cm}$. Although an increase in the floodplain width increased the above-mentioned difference, the main channel wall slope had no significant effects on it.

The CES model-estimated values of $\alpha$ and $\beta$ were, in general, higher than the experimental ones.

Before the flow depth reached a bankfull stage $(15 \mathrm{~cm})$, the difference between the experimental and estimated values of the discharge was reduced, but a flow-depth increase increased the difference in all series until it reached its maximum. An increase in the flow depth up to $25 \mathrm{~cm}$ reduced this difference, but a further increase kept the 
difference almost constant. A comparison of the estimated and experimental values showed that the CES model overestimated the flow discharge.

\section{Acknowledgments}

Thanking the reviewers for their thorough work and helpful comments, the author is also grateful to the Wallingford-HR Institute for providing the data for this research. The author declares that she has no known competing financial interests or personal relationships that could have appeared to influence the work reported in this paper.

This research did not receive any specific grant from the public, commercial, or not-for-profit funding agencies.

\section{List of Abbreviations}

$\begin{array}{ll}\text { CES } & \text { Conveyance Estimation System } \\ \text { FCF } & \text { Flood Channel Facility } \\ \text { SKM } & \text { Shino Knight Method } \\ \text { LDM } & \text { Lateral distribution method } \\ \text { SCM } & \text { Single Channel Method } \\ \text { EDM } & \text { Exchange Discharge Method } \\ \text { EVDM } & \text { Exchange vertical interface Discharge Model } \\ \text { RANS } & \text { Reynolds-averaged Navier-Stokes } \\ \text { NRMSE } & \text { Normalized Root Mean Square Error } \\ \text { MAPE } & \text { mean absolute percentage error } \\ \text { NITR } & \text { National Institute of Technology, Rourkela }\end{array}$

\section{References}

Bousmar D. (2002) Flow modeling in compound channels, momentum transfer between main channel and prismatic or non-prismatic floodplains, Thesis presented for the degree of Doctor in Applied Sciences, Universities catholique de Louvain.

Chow V. T. (1959) Open-channel hydraulics, [in:] Open-channel hydraulics, McGraw-Hill.

Devi K., Khatua K. K. (2019) Discharge prediction in asymmetric compound channels, Journal of Hydro-environment Research, 23, 25-39.

Devi K., Khuntia J. R., Khatua K. K. (2018) Depth-Averaged Velocity Distribution for Symmetric and Asymmetric Compound Channels, Proceedings of the International Conference on Microelectronics, Computing \& Communication Systems, Springer, Singapore, 281-292.

Ervine D. A., Babaeyan-Koopaei K., Sellin H. J. (2000) Two-dimensional Solution for Straight and Meandering Overbank Flows, Journal of Hydraulic Engineering, ASCE, 126 (9), 653-669.

Fenton J. D. (2005) On the energy and momentum principles in hydraulics, Proc. 31st Congress IAHR, Seoul, 625-636.

French R. H. (1987) Open-Channel Hydraulics, McGraw-Hill, Singapore, Second edition.

Hamidifar H., Omid M. H. (2013) Floodplain vegetation contribution to velocity distribution in compound channels, Journal of Civil Engineering and Urbanism, 3 (6), 357-361. 
Henderon F. M. (1966) Open channel flow, Macmilan Publishing Co, New York, United States of America.

Keshavarzi A., Hamidifar H. (2018) Kinetic energy and momentum correction coefficients in compound open channels, Natural Hazards, 92 (3), 1859-1869.

Keshavarzi A. (1993) Investigation of energy and momentum coefficients in compound channels, M.Sc. thesis, University of New South Wales, Australia.

Knight D. W., Shiono K., Pirt J. (1989) September. Prediction of depth mean velocity and discharge in natural rivers with overbank flow, Proceedings of the International Conference on Hydraulic and Environmental Modellling of Coastal, Estuarine and River Waters, Gower Publishing, 419-428.

Kolupaila S. (1956) Methods of determination of the kinetic energy factor, The Port Engineer, Calcutta, India, 5 (1), 12-18.

Li D., Hager W. H. (1991) Correction coefficients for uniform channel flow, Canadian Journal of Civil Engineering, 18 (1), 156-158.

Mohanty P. K., Khatua K. K. (2014) Estimation of discharge and its distribution in compound channels, Journal of Hydrodynamics, 26 (1), 144-154.

Mohanty P. K. (2013) Flow analysis of compound channels with wide flood plains Prabir (Doctoral dissertation).

Mohanty P. K., Dash S. S., Khatua K. K., Patra K. C. (2012) Energy and momentum coefficients for wide compound channels, River Basin Management, VII, 172, 87.

Moreta P. J. M., Lopez-Querol M. S. (2017) Numerical Modeling in Flood Risk Assessment: UK Case Study, Civil Engineering Research Journal, 3 (1), DOI: 10.19080/CERJ.2017.03.555601.

Nagy J., Kiss T., Fehérváry I., Vaszkó C. (2018) Changes in Floodplain Vegetation Density and the Impact of Invasive Amorpha fruticosa on Flood Conveyance, Journal of Environmental Geography, $11(3-4), 3-12$.

Parsaie A. (2016) Analyzing the distribution of momentum and energy coefficients in compound open channel, Modeling Earth Systems and Environment, 2 (1), 15.

Seckin G., Çă̆atay H., Çobaner M., Yurtal R. (2009a) Experimental investigation of kinetic energy and momentum correction coefficients in open channels, Scientific Research and Essay, 4 (5), 473-478.

Seckin G., Mamak M., Atabay S., Omran M. (2009b) Discharge estimation in compound channels with fixed and mobile bed, Sadhana, 34 (6), 923-945. , La Houille Blanche, (7), 793-802.

Shiono K., Knight D. W. (1988) Two-dimensional analytical solution for a compound channel, Proceedings of the 3rd international symposium on refined flow modeling and turbulence measurements, Proc. 3rd Int. Symp. on refined flow modeling and turbulence measurements, 503-510.

Shiono K., Knight D. W. (1990) Mathematical models of flow in two or multi stage straight channels, Proc. Int. Conf. on River Flood Hydraulics, Wiley New York, 229-238.

Shiono K., Knight D. W. (1991) Turbulent open-channel flows with variable depth across the channel, Journal of Fluid Mechanics, 222, 617-646.

Singh P. K. Banerjee S. Naik B., Kumar A., Khatua K. K. (2018) Lateral distribution of depth average velocity \& boundary shear stress in a gravel bed open channel flow, ISH Journal of Hydraulic Engineering, 1-15. 